\title{
The morphology of Chlamydia pneumoniae
}

\author{
N. MIYASHITA, Y. KANAMOTO* and A. MATSUMOTO
}

Department of Microbiology, Kawasaki Medical School, Kurashiki, Okayama 701-01, and *Division of Microbiology, Hiroshima Prefectual Institute of Public Health. Hiroshima 734, Japan

\begin{abstract}
Summary. The morphology of a recently isolated strain of Chlamydia pneumoniae, YK-41, was compared by electronmicroscopy with $C$. pneumoniae TWAR, Chlamydia trachomatis $\mathrm{L}_{2} / 434 / \mathrm{Bu}$ and Chlamydia psittaci Cal 10. The results showed that "pear-shaped" morphology was not typical of $C$. pneumoniae. Basic morphological features, such as surface projections and hexagonally arrayed, regular structures in the inside layer of the outer membrane of elementary bodies, were very similar in these strains. The structure of strain YK-41 was identical with that of $C$. trachomatis and $C$. psittaci, but the profiles of elementary bodies were different from those of $C$. pneumoniae TWAR strains.
\end{abstract}

\section{Introduction}

Chlamydiae are obligate intracellular parasites that differ from other prokaryotes in their growth cycle, in which there are two morphologically and functionally distinct cell types: the infectious, elementary body (EB) and the reproductive, reticulate body (RB). This unique growth cycle is common to all members of the genus Chlamydia, including C. trachomatis, C.psittaci, $C$. pneumoniae and $C$. pecorum. The latter two have recently been established as new Chlamydia species $^{1-3}$ and $C$. pneumoniae has been recognised as an important acute respiratory pathogen in man. ${ }^{4-6}$

Recently, Kanamoto et al. isolated a chlamydial strain, YK-41, from the nasopharynx of a 15-year-old man with acute bronchitis. ${ }^{7}$ The organism was highly reactive with a monoclonal antibody (MAb), RR-402 (Washington Research Foundation, Seattle, USA), directed against $C$. pneumoniae and with a genusspecific MAb (Cultureset ${ }^{\mathbb{k}}$, Ortho Diagnosis), but not with a MAb directed against $C$. trachomatis (MicroTrak ${ }^{\mathbb{R}}$, Syva Co.). The inclusions produced by strain YK-41 in HeLa 229 cells closely resembled those of $C$. psittaci in their morphology, absence of staining with iodine and expansion without compression of the host nucleus, indicating that it is a strain of $C$. pneumoniae. We have examined the morphology of strain YK-4l and compared it with that of recognised strains of $C$. pneumoniae, $C$. trachomatis and $C$. psittaci.

\section{Materials and methods}

\section{Organisms}

The chlamydiae used in this study were: $C$.

Received 13 April 1992; revised version received 1 Oct. 1992; accepted 30 Oct. 1992. pneumoniae strains YK-41, ${ }^{7}$ TW-183, AR-39 and AR$388 ; C$. psittaci $\mathrm{Cal} 10$; and C. trachomatis $\mathrm{L}_{2} / 434 / \mathrm{Bu}$. Strains TW-183, AR-39 and AR-388 were purchased from Washington Research Foundation, Seattle, USA and maintained in HeLa 229 cells as described previously. ${ }^{9}$ Strain $\mathrm{Cal} 10$ has been maintained for $>20$ years in our laboratory in monolayer or suspension cultures of the L 929 cell line. ${ }^{10}$ Strain $\mathrm{L}_{2} / 434 / \mathrm{Bu}$ was supplied by S. Yamasaki, National Institute of Health, Japan, and maintained in L 929 and McCoy cell lines.

\section{Infection and cultivation of the organisms}

HeLa 229 cells were the host cells throughout. After pre-treatment for $10 \mathrm{~min}$ with DEAE-dextran (final concentration $30 \mu \mathrm{g} / \mathrm{ml}$ ), cells were inoculated by centrifugation at $900 \mathrm{~g}$ for $60 \mathrm{~min}$ at room temperature and then incubated at $37^{\circ} \mathrm{C}$ in an atmosphere of $\mathrm{CO}_{2}$ $5 \%$ in Minimum Essential Medium (MEM; Nissui, Tokyo) containing cycloheximide $1 \mu \mathrm{g} / \mathrm{ml}$ and supplemented with heat-inactivated fetal calf serum (Gibco, USA) $10 \%$.

\section{Purification of EBS}

EBs of strain Cal 10 were purified from infected L 929 cells $48 \mathrm{~h}$ post-inoculation by the method of Tamura and Higashi. ${ }^{10}$

EBs of $C$. trachomatis $\mathrm{L}_{2} / 434 / \mathrm{Bu}$ and $C$. pneumoniae TW-183, AR-39, AR-388 and YK-41 were purified by a modification of the methods of Tamura and Higashi ${ }^{10}$ and Caldwell et al. ${ }^{11}$ Infected cells were collected $72 \mathrm{~h}$ post-inoculation and homogenised with a Teflon homogeniser. After centrifugation at $900 \mathrm{~g}$ for $10 \mathrm{~min}$ to remove cell debris, the supernate was layered on to a two-layered cushion-bottom layer, sucrose $50 \% \mathrm{w} / \mathrm{v}$ solution, top layer Urografin (3,5-diacetamido-2,4,6- 
triisobenzoic acid; Schering AG, Berlin/Bergkamen, Germany) $30 \% \mathrm{v} / \mathrm{v}$ in $30 \mathrm{~mm}$ Tris- $\mathrm{HCl}$ buffer, $\mathrm{pH} \mathrm{7.3-and} \mathrm{then} \mathrm{centrifuged} \mathrm{at} 8000 \mathrm{~g}$ for $60 \mathrm{~min}$. The pellet and turbid bottom layer were suspended in sucrose-phosphate-glutamate (SPG: sucrose 7.5\%, $\mathrm{KH}_{2} \mathrm{PO}_{4} \quad 0.052 \%, \quad \mathrm{Na}_{2} \mathrm{HPO}_{4} \cdot 2 \mathrm{H}_{2} \mathrm{O} \quad 0.1529 \%$, glutamic acid $0.072 \%$ ), and then centrifuged at $12000 \mathrm{~g}$ for $30 \mathrm{~min}$. After resuspending the pellet in SPG, the suspension was layered on a continuous Urografin gradient column $(40-52 \% \mathrm{v} / \mathrm{v})$ and centrifuged at $8000 \mathrm{~g}$ for $60 \mathrm{~min}$. Two distinct bands were formed in the gradient column. The presence of a number of complete EBs was confirmed in the lower band by electronmicroscopy. After washing with SPG, the EBs were suspended in SPG and stored at $-70^{\circ} \mathrm{C}$ until required.

\section{Preparation of EB outer membranes}

Outer membranes were isolated from the purified EBs by a modification of a previously described method. ${ }^{12}$ The purified EB suspension $(4 \mathrm{ml})$ in the Tris buffer was mixed with $6 \mathrm{~g}$ of glass beads $(0.1 \mathrm{~mm}$ in diameter; $\mathbf{M}$ and $\mathbf{S ~ I n s t r u m e n t s ~ I n c . , ~ O s a k a , ~ J a p a n ) ~}$ and shaken vigorously on a Cell Mill shaker (Edmund Bühler, Tübingen, Germany) at $70 \mathrm{CPS}$ for $4 \mathrm{~min}$. After centrifugation at $10000 \mathrm{~g}$ for $60 \mathrm{~min}$ on a sucrose cushion (sucrose $15 \% \mathrm{w} / \mathrm{v}$ ), the pellet was suspended in $0.2 \mathrm{M}$ Tris- $\mathrm{HCl}$ buffer ( $\mathrm{pH} 7.4$ ) containing $\mathrm{MgCl}_{2}$ $5 \mathrm{mg} / \mathrm{ml}$ and treated with a mixture of DNAase and RNAase (final concentration $20 \mu \mathrm{g} / \mathrm{ml}$, each) at $37^{\circ} \mathrm{C}$ for $60 \mathrm{~min}$. This was followed by trypsin treatment $(50 \mu \mathrm{g} / \mathrm{ml})$ at $37^{\circ} \mathrm{C}$ for $60 \mathrm{~min}$ and finally with sodium dodecyl sulphate (SDS) $0.125 \%$ at $37^{\circ} \mathrm{C}$ for $10 \mathrm{~min}$. After washing twice, the outer membranes were suspended in distilled water and stored at $-70^{\circ} \mathrm{C}$ until required.

\section{Electronmicroscopy}

To examine the morphology of organisms in situ, infected cells were collected at 24, 36, 48, 60 and $72 \mathrm{~h}$ post-inoculation by centrifugation at $300 \mathrm{~g}$ for $3 \mathrm{~min}$ and thin sections were prepared as reported previously. ${ }^{13}$

For shadowcast preparations, EBs or outer membranes were mounted on a specimen grid. ${ }^{14}$ For negative staining, outer membranes were mounted on a carbon covered grid and placed on to a droplet of phosphotungstic acid (PTA) $1 \%$ solution. To prepare freeze-replicas, infected cells were pelleted by centrifugation, fixed with glutaraldehyde $2.5 \%$ and then impregnated with glycerol $40 \%$ to prevent morphological damage by ice crystal formation. After freezing in liquid nitrogen, replicas were prepared in a Balseres 3AF 301 freeze-replica apparatus as described previously. ${ }^{15}$

Specimens were examined with a Hitachi H-500 or JEM-2000 transmission electronmicroscope at 75 or $80 \mathrm{kV}$.
To observe the projections on EB surfaces of $C$. pneumoniae strains, the purified EBs were mounted on a nickel grid without a supporting membrane and doubly fixed with glutaraldehyde and $\mathrm{OsO}_{4}$ fixatives. Specimens were additionally treated with tannic acid $2 \%$ to stabilise the morphology of the projections, as reported previously. ${ }^{16}$ Specimens were dehydrated in an ethanol series, dried in a Hitachi HCP-1 critical point dryer and coated with Pt-palladium alloy in a Hitachi E-102 Ion Sputter unit. Specimens were examined with a Hitachi S-570 scanning electronmicroscope at $25 \mathrm{kV}$.

\section{Determination of the spacing of regular structure by Fourier transformation}

We have previously used optical transformation to determine the spacing of the hexagonal structure in the EB outer membrane with laser beams, by which the regular structure was transformed into a diffraction image. ${ }^{17}$ In this study, the diffraction image was obtained by the formula programmed in an IBAS 2000 image analysis system (Zeiss, Germany). Briefly, the information in a micrograph was put into the image memory of the system through a camera and the Fourier integral was calculated in the computer system, so that the original image in the micrograph was displayed as a diffraction pattern. By measurement of the distance from the origin of the transform (frequency 0 ) to the diffraction spots, the average spacing of the regular structure was calculated. In some cases, the images were reconstituted from the selected spots in the diffraction pattern by the inverse Fourier transform to obtain a clear image without the "noise" present in the original micrographs.

\section{Results}

\section{Morphology of $Y K-41 E B$ in thin sections and shadowcast preparations}

The chlamydial bodies in in-situ inclusions of the $C$. pneumoniae strains $60 \mathrm{~h}$ post-inoculation are shown in figs. 1a-d. A number of EBs and RBs are seen in the inclusions, and all are free from glycogen particles. There is no distinct difference in the RB morphology of the different strains. However, the EBs of strain YK-41 (fig. 1d) were round and there was no clear difference between the EB morphology of YK-41, $C$. psittaci and $C$. trachomatis strains. Each strain YK-41 EB had a dense nucleus at an eccentric site in the cell body and a narrow periplasmic space. In contrast, the EBs of the TWAR strains had a wide periplasmic space limited by a wavy outer membrane, forming "pear-shaped" profiles, and the nucleus in each EB was at an eccentric site in the cell body which appeared to be enclosed tightly within a cytoplasmic membrane (figs. 1a-c). A similar morphological difference was seen when the purified EBs were dried in air and 

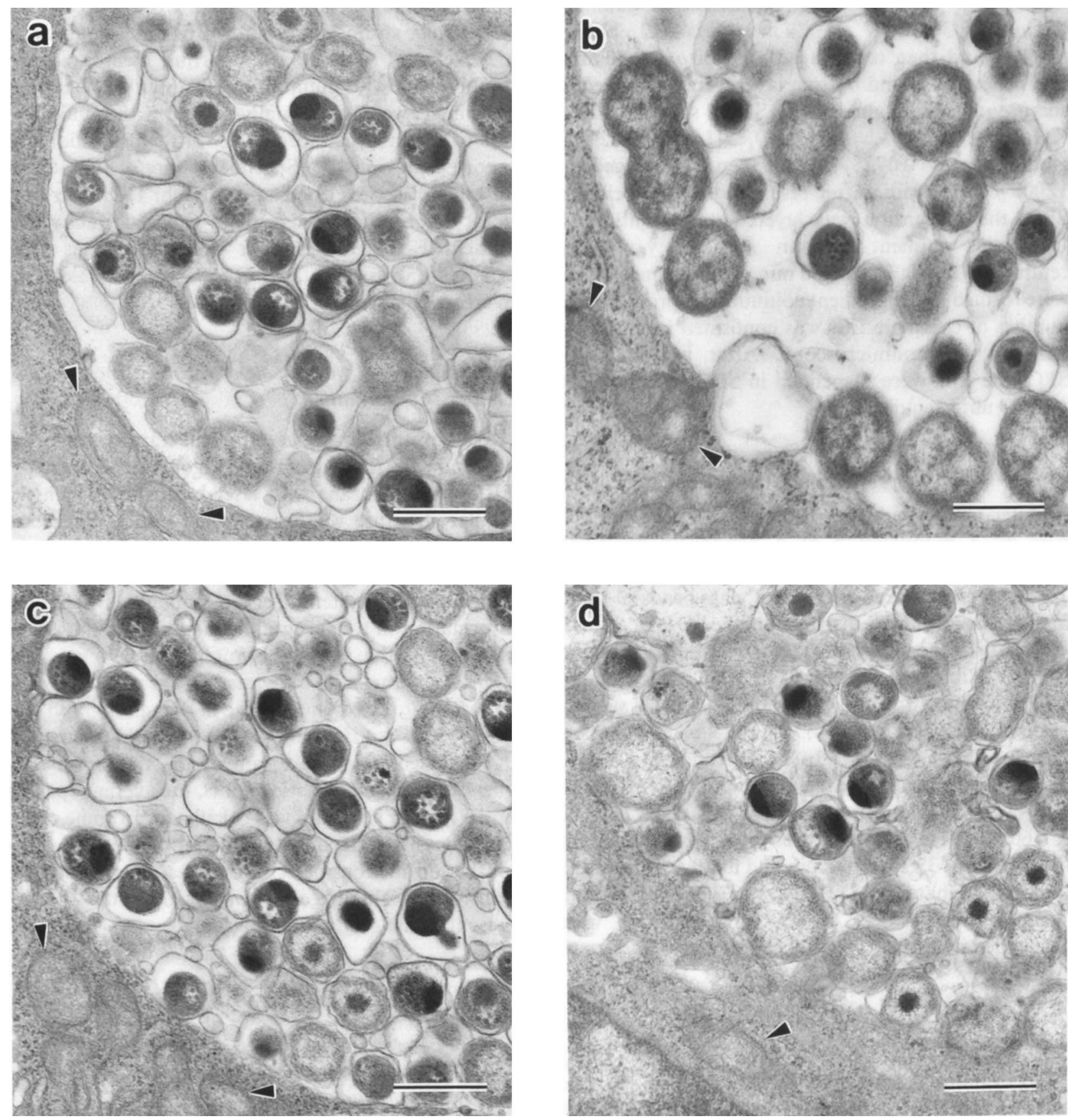

Fig. 1. Thin sections of $C$. pneumoniae inclusions in HeLa 229 cells $72 \mathrm{~h}$ post-inoculation. a, strain TW-183; b, AR-39; c, AR-388; d, YK-41. Strain YK-41 EBs are round; those of strains TW-183, AR-39 and AR-388 are enclosed by a wavy outer membrane and are "pear-shaped" in profile. There is no difference in the morphology of RBs. Arrowheads indicate mitochondria located close to inclusions, but not associated with the inclusion membrane. Bars, $1 \mu \mathrm{m}$.

shadowcast (fig. 2). The EBs of the Cal 10 and $\mathrm{L}_{2} / 434 / \mathrm{Bu}$ strains (figs. $2 \mathrm{a}$ and $\mathrm{b}$ ) had a round "fried egg"-like shape indistinguishable from that of the EBs of strain YK-41 (fig. 2d). However, the EBs of strain TW-183 had a "pear-shaped" morphology with a wide outer-membrane area even by the air-drying method (fig. 2c). These results led us to conclude that the EB morphology of EB of strain YK-41 was identical to that of C. psittaci and C. trachomatis EBs, but different from that of the EBs of TWAR strains of $C$. pneumoniae. Further observations on the distribution of host mitochondria in the cells infected with the $C$. pneumoniae strains revealed no mitochondrial association with the inclusion membrane in strain YK41 or the TWAR strains examined (fig. 1, arrowheads).

\section{Surface projections and related structures}

To examine the internal structures of the EB envelope and the inclusion membrane, freeze-replicas of the inclusion-bearing cells were prepared $60 \mathrm{~h}$ postinoculation. As shown in fig. 3a, the cleaved faces of the EBs and RBs were exposed as convex or concave faces in the replica membrane. The button structures (B structures) ${ }^{15}$ or the craters ${ }^{18}$ were clearly seen on a limited area of the concave faces (fig. 3a, arrowheads). 

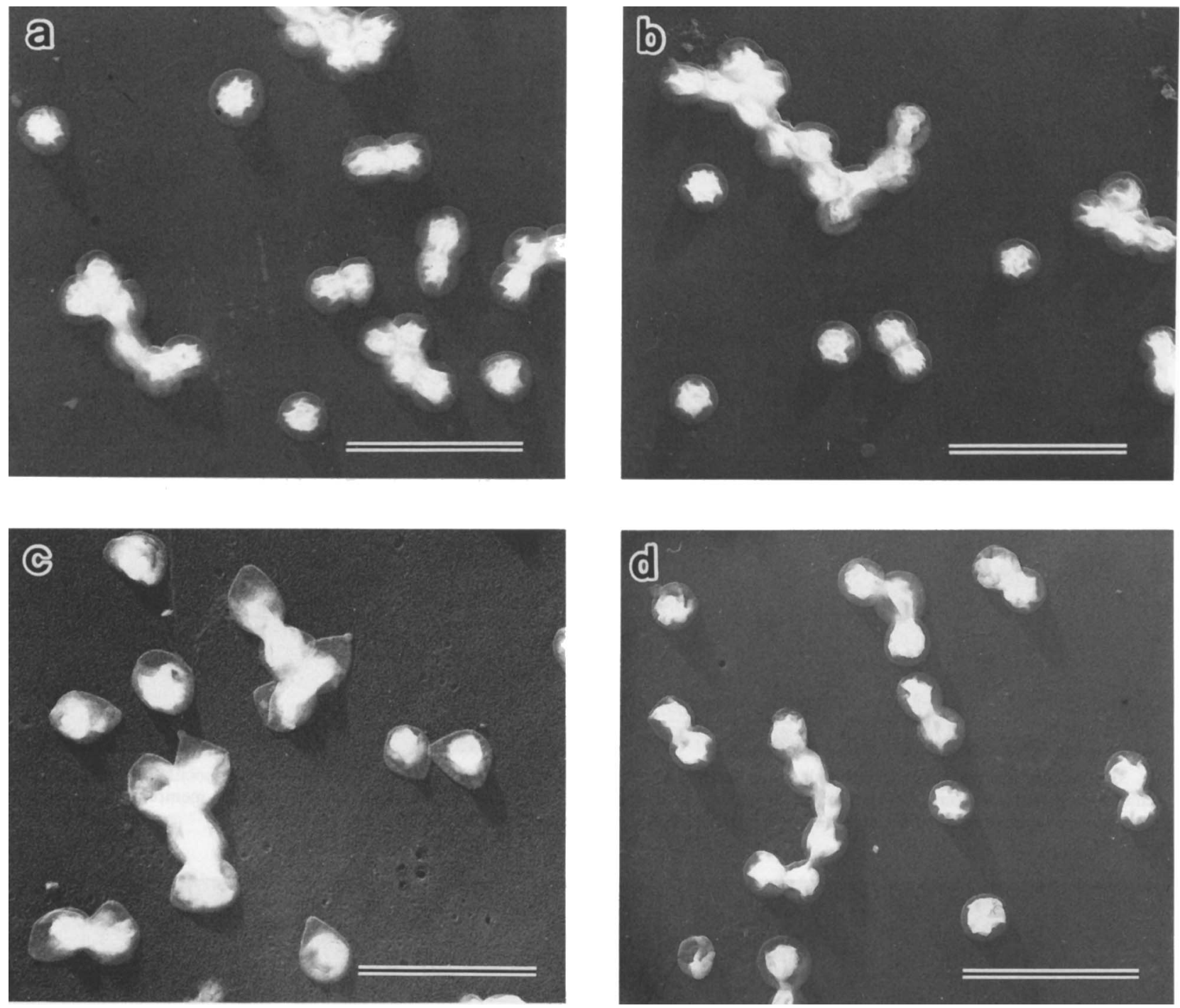

Fig. 2. Purified EBs in shadowcast preparations. EBs were air-dried, mounted on specimen grids and shadowcast with Pt-palladium alloy. a, C. psittaci Cal 10 ; b, C. trachomatis $\mathrm{L}_{2} / 434 / \mathrm{Bu} ; \mathrm{c}$, C. pneumoniae TW-183; d, C. pneumoniae $\mathrm{YK}-41$. EBs of strains Cal $10, \mathrm{~L}_{2} / 434 / \mathrm{Bu}$ and YK-41 have a "fried egg" shape, whereas EBs of strain TW-183 are pleomorphic but "pear-shaped" after air-drying. Bars, $1 \mu \mathrm{m}$.

The dimension of each $B$ structure, the spacing between neighbouring $\mathrm{B}$ structures and the arrangement in the groups were similar to those in the EBs of strain Cal 10 reported previously. ${ }^{18}$ When the inclusion membrane was replicated, many fine particles grouped together within several areas were frequently observed on the convex face of the inclusion membrane (fig. $3 b$ ). In each group, the arrangement of the particles was roughly hexagonal with a spacing of $30-50 \mathrm{~nm}$. Identical particles were detected on TWAR strain inclusions (data not shown). The morphology of the particles was similar to that seen on the inclusion membrane of strain Cal $10 .{ }^{19}$ These results strongly suggested the presence of the projections on the surface of the strain YK-41 EBs and RBs in inclusions in situ. To confirm the presence of projections on the EBs of YK-41 and TWAR strains, purified EBs were dried by the critical point drying method and examined by scanning electronmicroscopy. Projections were observed on a limited area of the EB surface of $C$. pneumoniae strains (fig. 4). The EBs of the TWAR strains (figs. 4a-c) were irregular in shape and this correlated with the presence of a wide periplasmic space, whereas the EBs of strain YK-41 were globular in shape (fig. 4d). The number of projections on the EBs of each strain was not determined.

\section{Regular structure in the outer membranes}

In a preliminary experiment, it was noted that the EB outer membranes of TWAR strains were broken into small pieces by treatment with SDS $0.5 \%$, whereas those of the EB of strain Cal 10 were not. This suggested a difference in outer-membrane stability between $C$. pneumoniae and $C$. psittaci. Therefore, the SDS concentration was reduced to $0 \cdot 125 \%$ for the isolation of outer membranes of TWAR and YK-41 strains.

When negatively stained, the hexagonally arrayed structures were clearly seen in all specimens (figs. 5al-d1). The dimension of the structures appeared to be similar in all strains examined. Such regular 

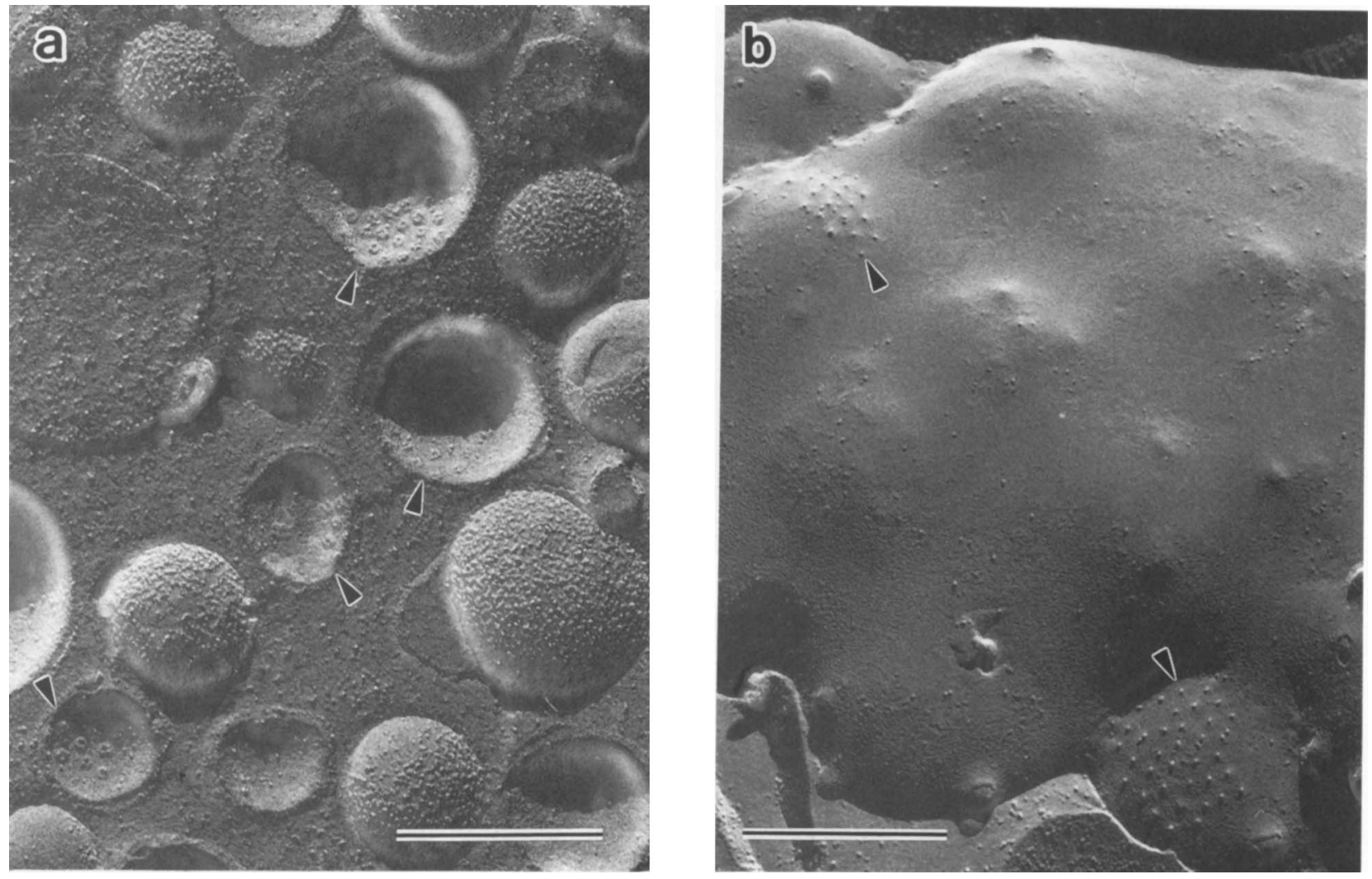

Fig. 3. Freeze replica images of in-situ chlamydial bodies and inclusion membranes of strain YK-41 $60 \mathrm{~h}$ post-inoculation. a, chlamydial bodies are cleaved into convex or concave faces, and many B structures are present (arrowheads); $\mathbf{b}$, the inclusion membrane is exposed by cleavage. Two groups of particles arrayed hexagonally are present (arrowheads). Bars, $0.5 \mu \mathrm{m}$.
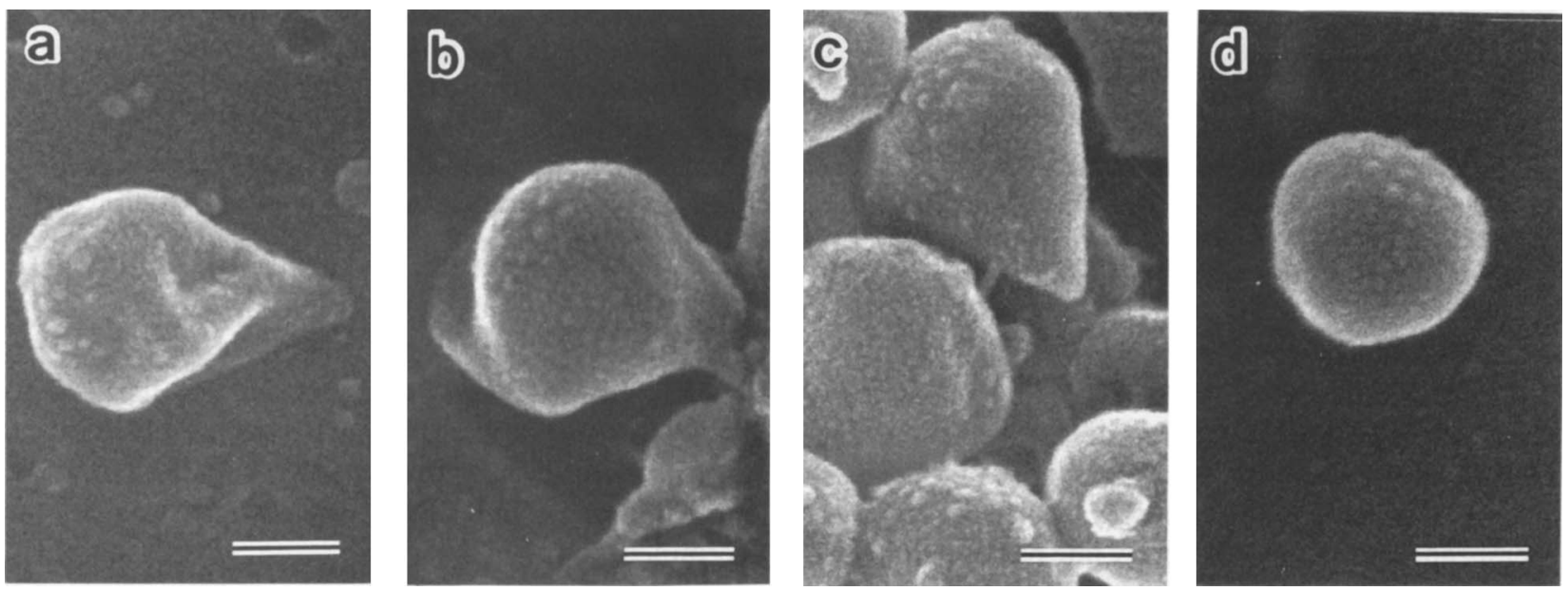

Fig. 4. Scanning electronmicroscopic images of purified EBs of $C$. pneumoniae strains: a, TW-183; b, AR-39; c, AR-388; d, YK-41. All EBs have hexagonally arrayed projections on a limited area of the surface. EBs of strain YK-41 are globular, those of other strains are pleomorphic. Bars, $100 \mathrm{~nm}$.

structures were also seen in the shadowcast preparations only on the inner surface, which was exposed by the disintegration of the outer membrane. These results agree with those reported previously, ${ }^{20}$ indicating that the inner surface of the EB outer membrane of the $C$. pneumoniae strains is also composed of hexagonally arrayed structures.

To determine the spacing of the hexagonal structure, a computer Fourier transform was carried out. An example is shown in fig. 6. Fourier transform of a fragment of the outer membrane of strain YK-41 (fig. 6a) resulted in the diffraction spots shown in fig. $6 \mathrm{~b}$.
These show three different structural units of about 176,90 and $50 \AA$, respectively. Reconstitution by the inverse Fourier transform for the innermost spots (176 Å) produced a clear hexagonal pattern (fig. 6c). The periodicity in the outer membrane of all strains used in the present study was measured in this way and the results are summarised in the table. The average periodicity of the hexagonal structure of the TWAR strains closely resembled one another, whereas that of the Cal 10 and YK-41 strains tended to be a little larger. The localisation of the outer two structural units (90 and $50 \AA$ A) was not determined. 


\section{a1}
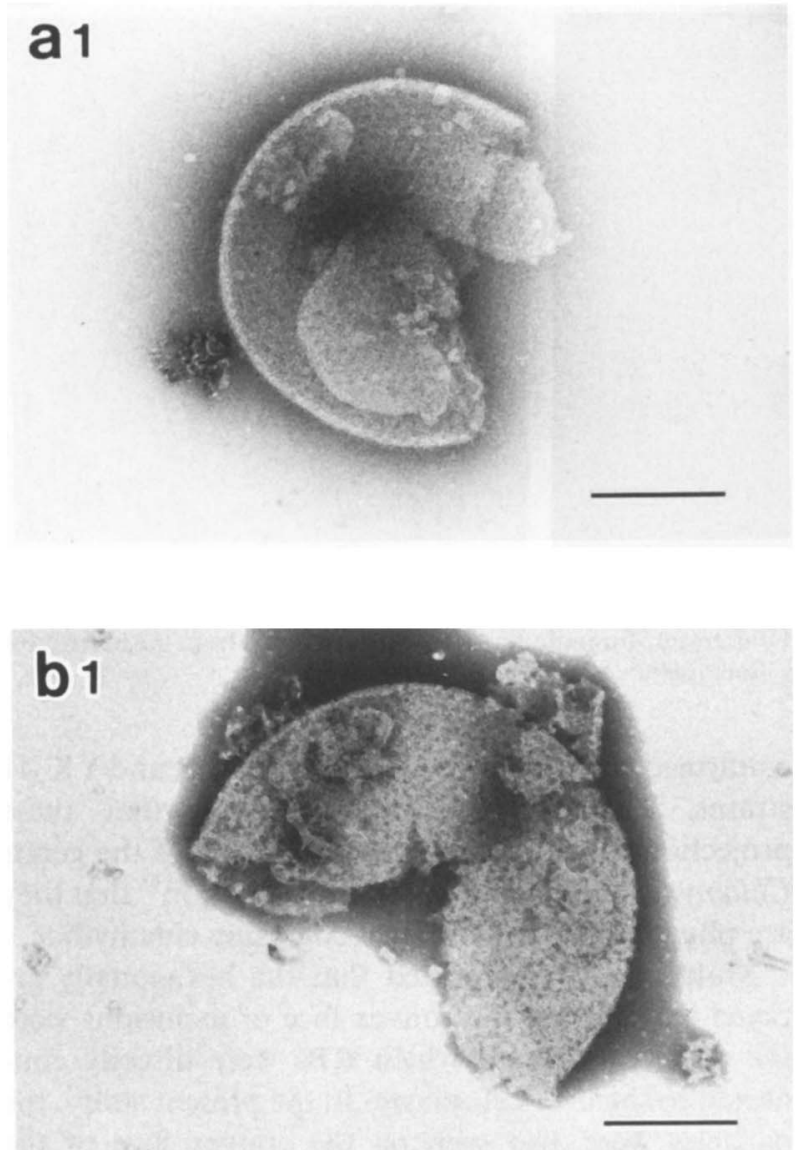

C1

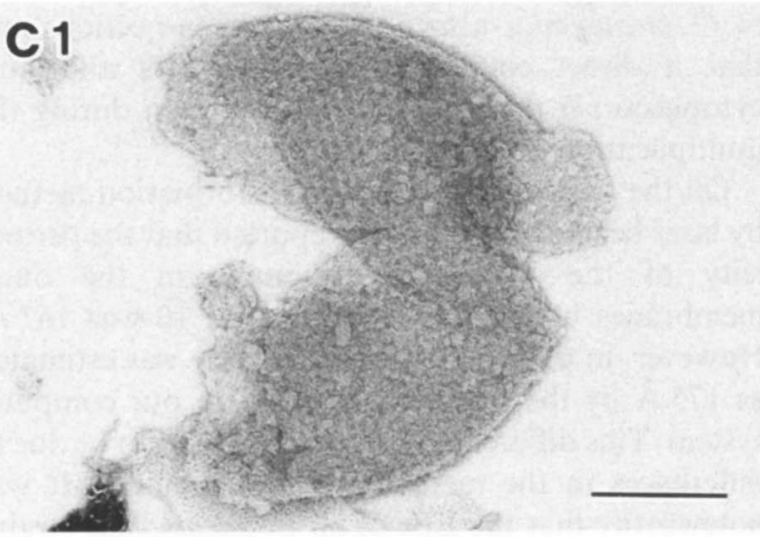

\section{d 1}

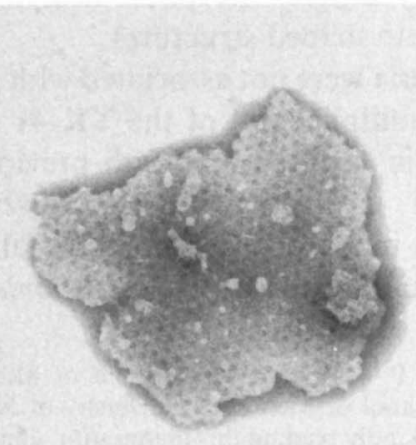

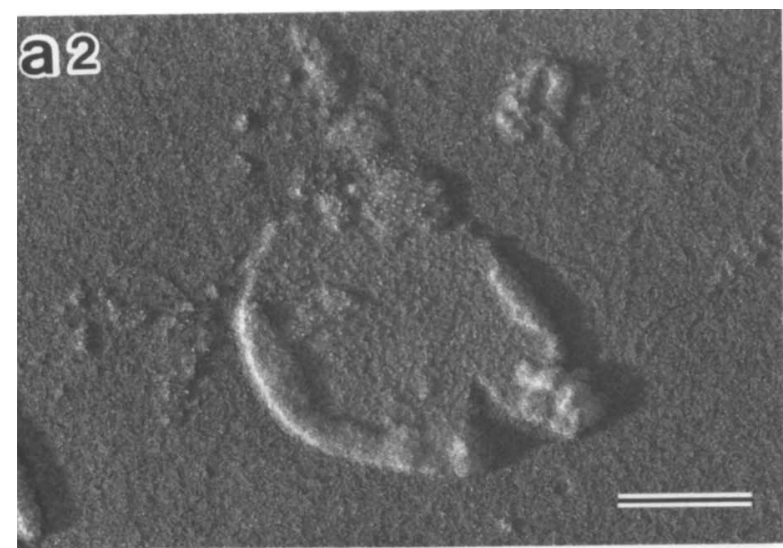
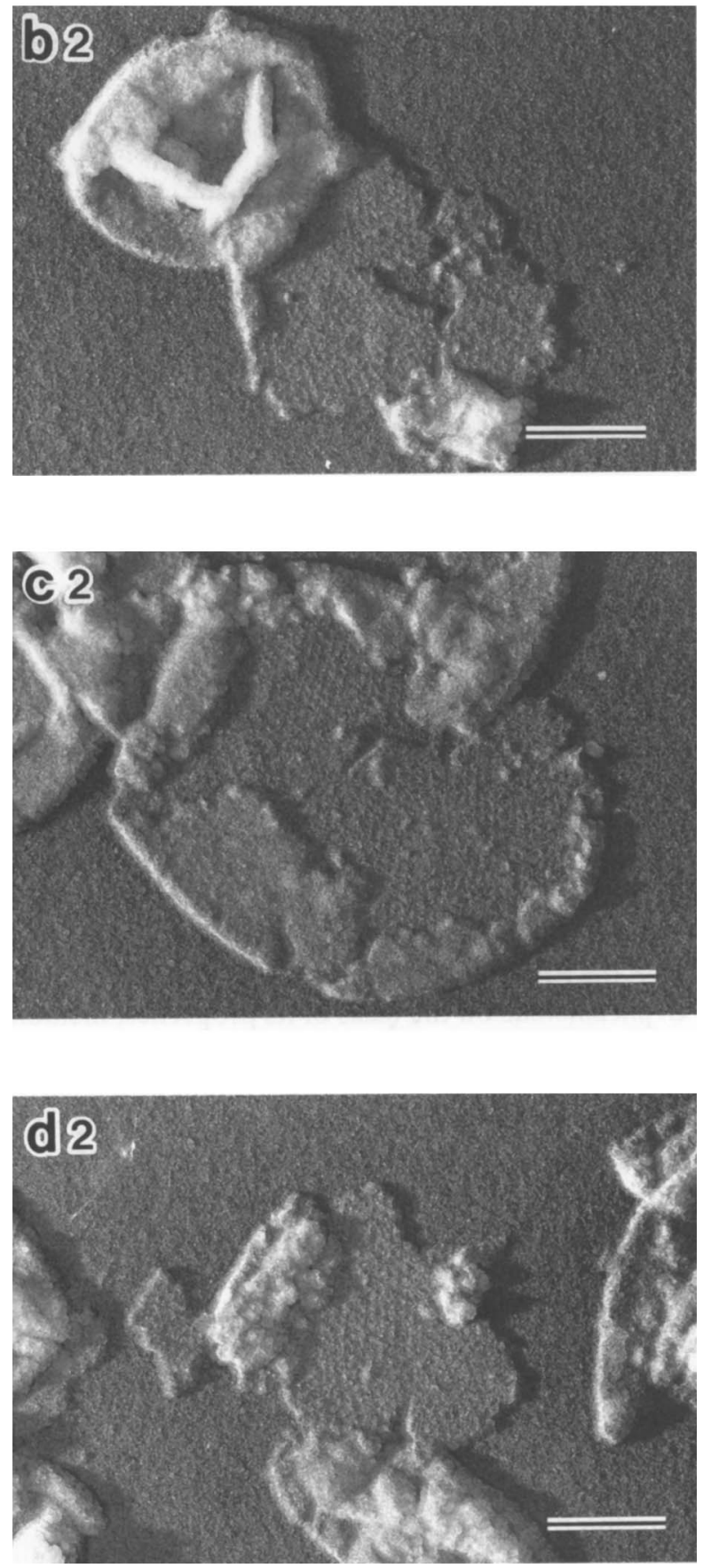

Fig. 5. Negatively stained and shadowcast images of EB outer membranes isolated from purified EBs of $C$. pneumoniae. a, strain TW-183; b, AR-39; v, AR-388; d-YK-41. The hexagonal structure seen throughout outer membranes (a1-d1) is seen only on the inner surface (a2-d2). The periodicity of the hexagonal structures of different strains appears to be very similar. Bars, $200 \mathrm{~nm}$. 

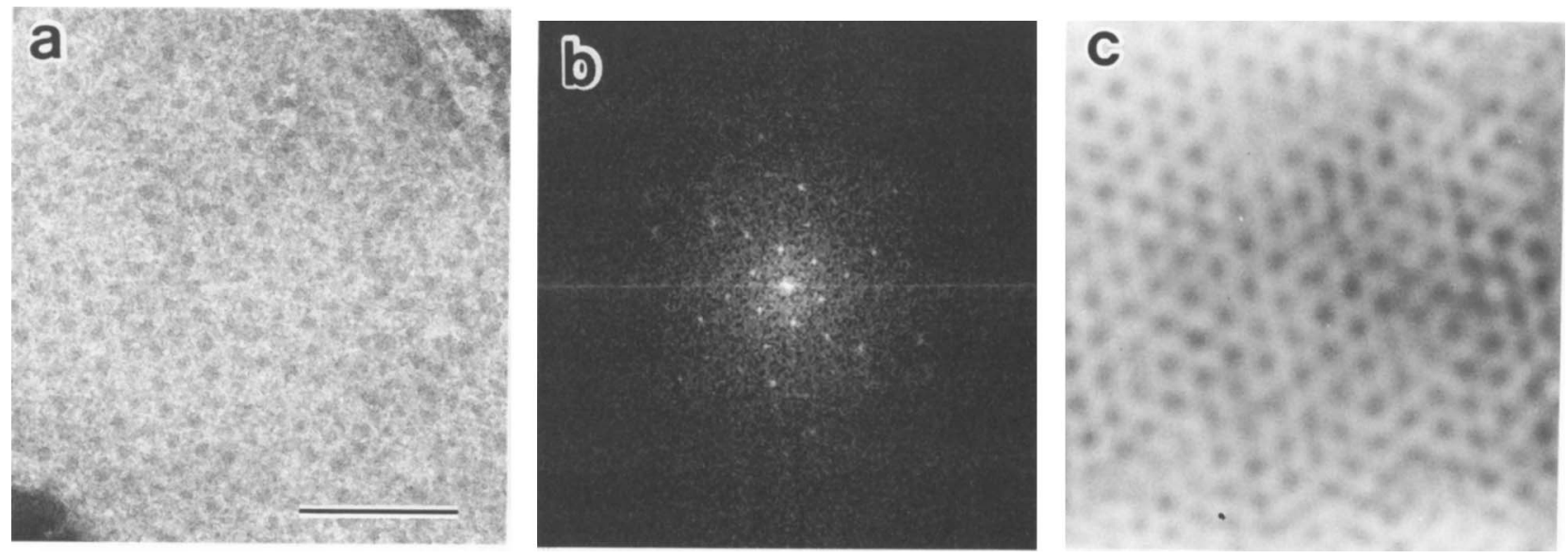

Fig. 6. Image processing of the hexagonal structures in outer membrane of strain YK-41 EB. a, original, negatively stained outer membrane; b, diffraction spots obtained from the original micrograph shown in a by Fourier transformation; c, image reconstituted from the inner-most six spots of the diffraction shown in $\mathbf{b}$ by inverse Fourier transformation. Bar, $100 \mathrm{~nm}$.

Table. Comparison of the periodicity between $C$. psittaci and C. pneumoniae strains*

\begin{tabular}{llc}
\hline Species & $\begin{array}{c}\text { Strain } \\
\text { no. }\end{array}$ & $\begin{array}{c}\text { Mean (SD) periodicity } \\
(\AA)\end{array}$ \\
\hline C.psittaci & Cal 10 & $175(8)$ \\
C. pneumoniae & TW-183 & $172(7)$ \\
& AR-39 & $174(8)$ \\
& AR-388 & $172(7)$ \\
& YK-41 & $176(6)$ \\
\hline
\end{tabular}

*Periodicity as measured from centre to centre by Fourier transform.

\section{Discussion}

The morphology of $C$. pneumoniae strains TW-183 and AR-39 has been compared with that of $C$. trachomatis $\mathrm{L}_{2} / 434 / \mathrm{Bu}$ and $C$. psittaci $\mathrm{Cal} 10$ and it was shown that the EBs of the $C$. pneumoniae strains were "pear-shaped" with a large periplasmic space, whereas those of $C$. trachomatis and $C$. psittaci were round as seen in thin sections. ${ }^{21}$ Such a morphological difference was confirmed in the present study. However, the EBs of strain YK-41, which was recognised as $C$. pneumoniae on the basis of its properties, including reactivity with $C$. pneumoniae-specific MAb RR-402 and stainability with iodine, were found with a narrow periplasmic space in sections and shadowcast preparations. Carter et $a l .^{22}$ demonstrated that the morphology of EBs of $C$. pneumoniae IOL-207 also appeared round in thin sections and were indistinguishable from those of the $C$. trachomatis and C. psittaci. Similarly, Popov et al. ${ }^{23}$ demonstrated round EBs in thin sections of $C$. pneumoniae Kajaani6. These results, together with those presented here, strongly suggest that the "pear-shaped" profiles of EBs in thin sections are not common in $C$. pneumoniae and that the pear-shape is not a morphological criterion for recognising $C$. pneumoniae.

The presence of the projections on EBs of many strains of $C$. psittaci and of $C$. trachomatis has been shown by electronmicroscopy. ${ }^{24-26}$ This was confirmed, and not only on EBs of TWAR and YK-41 strains. The results strongly suggest that these projections are common to all members of the genus Chlamydia and this supports the suggestion ${ }^{25}$ that they are phenotypic markers for recognising chlamydiae.

Matsumoto $^{24,27}$ reported that the hexagonally arrayed particles on the convex face of inclusions were the projections, with which RBs were directly connected to the host cytoplasm. In the present study, the particles were also seen on the convex face of the replicated inclusions of the $C$.pneumoniae TWAR and YK-41 strains. Therefore, it is very likely that the RBs of $C$. pneumoniae also possess these projections and that a direct connection between RBs and host cytoplasm via the projections is common during the multiplication of chlamydiae.

On the basis of the optical transformation method by laser beam, Matsumoto ${ }^{17}$ reported that the periodicity of the hexagonal structures in the outer membranes of the EBs of strain Cal 10 was $167 \AA$. However, in this study, this periodicity was estimated as $175 \AA$ by the Fourier transform in our computer system. This difference of $c .5 \%$ appeared to be due to differences in the methods of measurement. It was noteworthy that the EBs of all $C$. pneumoniae strains examined possessed hexagonal structures with a similar periodicity despite morphological differences of round and pear-shaped structures.

Mitochondria were not associated with inclusions in situ during multiplication of the YK-41 and TWAR strains. This is in agreement with previous results. ${ }^{13}$ Therefore, it is likely that the absence of the mitochondria-inclusion association is also common during the multiplication of $C$. pneumoniae.

We thank Dr P. B. Wyrick, Department of Microbiology and Immunology, School of Medicine, University of North Carolina, Chapel Hill, for kindly reading this manuscript, and Dr Y. Kondo, Department of Physics, Kawasaki Medical School for his help with the Fourier transform. We also thank K. Yamane, K. Uehira and T. Suda of the Electron Microscope Center, and S. Ohmori and H. Murakami, of the Department of Microbiology, Kawasaki Medical School for their technical assistance. This work was supported in part by Research Grant (1-512), Kawasaki Medical School. 


\section{References}

1. Grayston JT, Kuo CC, Campbell LA, Wang SP. Chlamydia pneumoniae sp. nov. for Chlamydia sp. strain TWAR. Int $J$ Syst Bacteriol 1989; 39: 88-90.

2. Grayston JT. Chlamydia pneumoniae, strain TWAR. Chest 1989; 95: 664-669.

3. Fukushi H, Hirai K. Proposal of Chlamydia pecorum sp. nov. for Chlamydia strains derived from ruminants. Int J Syst Bacteriol 1992; 42: 306-308.

4. Grayston JT, Kuo CC, Wang SP, Altman J. A new Chlamydia psittaci strain, called TWAR, isolated in acute respiratory tract infections. $N$ Engl J Med 1986; 315: 161-168.

5. Marrie TJ, Grayston JT, Wang SP, Kuo CC. Pneumonia associated with the TWAR strain of Chlamydia. Ann Intern Med 1987; 106: 507-511.

6. Grayston JT, Campbell LA, Kuo CC et al. A new respiratory tract pathogen: Chlamydia pneumoniae, strain TWAR. $J$ Infect Dis 1990; 161: 618-625.

7. Kanamoto Y, Sakano T. Isolation of Chlamydia pneumoniae from a patient with acute bronchitis. JJpn Assoc Infect Dis 1992; 66: 637-642. (In Japanese.)

8. Kuo CC, Chen HH, Wang SP, Grayston JT. Identification of a new group of Chlamydia psittaci strains called TWAR. $J$ Clin Microbiol 1986; 24: 1034-1037.

9. Kuo CC, Wang SP, Wentworth BB, Grayston JT. Primary isolation of TRIC organisms in HeLa 229 cells treated with DEAE-dextran. $J$ Infect Dis $1972 ; 125$ : 665-668.

10. Tamura A, Higashi N. Purification and chemical composition of meningopneumonitis virus. Virology 1963; 20: 596-604.

11. Caldwell HD, Kromhout J, Schachter J. Purification and partial characterization of the major outer membrane protein of Chlamydia trachomatis. Infect Immun 1981 ; 31 : 1161-1176.

12. Tamura A, Matsumoto A, Manire GP, Higashi N. Electron microscopic observations on the structure of the envelopes of mature elementary bodies and developmental reticulate forms of Chlamydia psittaci. J Bacteriol 1971; 105: $355-360$.

13. Matsumoto A, Bessho H, Uehira K, Suda T. Morphological studies of the association of mitochondria with chlamydial inclusions and the fusion of chlamydial inclusions. $J$ Electron Microsc 1991; 40: 356-363.

14. Matsumoto A, Higashi N, Tamura A. Electron microscope observations on the effects of polymixin B sulfate on cell walls of Chlamydia psittaci. J Bacteriol 1973; 113: 357-364.
15. Matsumoto A. Fine structures of the cell envelopes of Chlamydia organisms as revealed by freeze-etching and negative staining techniques. $J$ Bacteriol 1973; 116: $1355-1363$

16. Matsumoto A. Electron microscopic observations of surface projections and related intracellular structures of Chlamydia organisms. J Electron Microsc 1981; 30: 315-320.

17. Matsumoto A. Recent progress of electron microscopy and its development in future; from a study of the obligate intracellular parasites, Chlamydia organisms. $J$ Electron Microsc 1979; 28 Suppl: S57-S64.

18. Louis C, Nicolas G, Eb F, Lefebvre JE, Orfila J. Modifications of the envelope of Chlamydia psittaci during its developmental cycle: freeze-fraction study of complementary replica. J Bacteriol 1980; 141 : 868875 .

19. Matsumoto A. Electron microscopic observations of surface projections on Chlamydia psittaci reticulate bodies. $J$ Bacteriol 1982; 150: 358-364.

20. Matsumoto A, Manire GP. Electron microscopic observations on the fine structure of cell walls of Chlamydia psittaci. $J$ Bacteriol 1970; 104: 1332-1337.

21. Chi EY, Kuo CC, Grayston JT. Unique ultrastructure in the elementary body of Chlamydia sp. strain TWAR. $J$ Bacteriol 1987; 169: 3757-3763.

22. Carter MW, Al-Mahdawi SAH, Giles IG, Treharne JD, Ward ME, Clarke IN. Nucleotide sequence and taxonomic value of the major outer membrane protein gene of Chlamydia pneumoniae IOL-207. J Gen Microbiol 1991; 137: 465-475.

23. Popov VL, Shatkin AA, Pankratova VN et al. Ultrastructure of Chlamydia pneumoniae in cell culture. FEMS Microbiol Lett 1991; 84: 129-134.

24. Matsumoto A. Structural characteristics of chlamydial bodies. In: Barron AL (ed) Microbiology of Chlamydia. Florida, USA, CRC Press. 1988: 21-45.

25. Gregory WW, Gardner M, Byrne GI, Moulder JW. Arrays of hemispheric surface projections on Chlamydia psittaci and Chlamydia trachomatis observed by scanning electron microscopy. J Bacteriol 1979; 138 : 241-244.

26. Matsumoto A, Bessho H. Surface projections of elementary bodies of Chlamydia strains. In: Imura T, Maruse S, Suzuki T (eds) Electron microscopy 1986. Tokyo, Marzen. 1986: 3349-3350.

27. Matsumoto A. Isolation and electron microscopic observations of intracytoplasmic inclusions containing Chlamydia psittaci. J Bacteriol 1981; 145: 605-612. 